\title{
THE INTRINSIC PULSATION RATES OF FRAG- MENTS OF THE EMBRYONIC CHICK HEART
}

\author{
ALEXANDER BARRY \\ Anatomy Department of the University of Michigan, Ann Arbor ${ }^{4}$, and The Biological \\ Laboratories of Harvard University, Cambridge, Massachusetts
}

FIVE FIGURES

\section{INTRODUCTION}

It has been shown previously that the pulsation frequency of the heart of the embryonic chick increases rapidly during: the first hours after its activity begins. Ordinarily the first heart beats occur at about the twenty-ninth to the thirtieth hours of incubation. During the next few hours the acceleration is at first rapid, but thereafter declines. By the fiftieth hour of incubation the rate of acceleration (not the rate of beating) has become markedly less (Barry, '40). The present investigation is the first step in an attempt to determine the causes of this phenomenon. Since the heart is spontaneously rhythmic, its rate must be fundamentally determined by the inherent pulsation frequency of some intrinsic pacemaking mechanism. This basic rate may be altered by secondary factors in the immediate environment of the myocardium. The data presented here establish the inherent rhythmicity of the myocardium at various cardiac levels and at various ages of the embryonic heart. Only after such information is available can the effect of secondary modifying factors be evaluated.

The heart is formed by the successive addition of myocardial tissue to its caudal end (Patten, 22). Since each

\footnotetext{
1 The construction of the apparatus used in these experiments was made possible by a grant from the Faculty Research Fund of the University of Michigan.
} 
chamber thus formed has a higher intrinsic rate than the parts of the heart previously established, it becomes in its turn, the pacemaker. The inherent frequency of pulsation of the chambers of the young cardiac tube have been measured and described since the time of Pickering (1897). The early work has been reviewed and discussed by Patten and Kramer ('33). It will therefore, be sufficient here to confine our attention to their paper and some of the other recent contributions that have a direct bearing on the present investigation. Johnstone ('24) showed that the cephalo-caudal gradient in the rates of pulsation of the various chambers of the heart could be demonstrated both by transsection and by ligation. Cohn ('25) studied, in culture, the rates of pulsation of explants from several regions of the embryonic heart. His results indicated that the cephalo-caudal gradient in cardiac rate was present within the individual chambers. He showed also that the more anterior regions of the hearts of older embryos lost their automaticity.

More recently Copenhaver ('39) has reported a very complete series of sectioning experiments on the heart of the developing Amblystoma punctatum. His results clearly demonstrate a gradient along the axis of the heart both between and within its several chambers. He further shows that after a region of the heart has lost its pacemaker function, its inherent rate of pulsation decreases gradually.

The present fragmentation experiments on the heart of the embryonic chick were undertaken to study the intrinsic rates of the several regions of the myocardium during the first few days of its development. Particular care was taken to insure that these rates be measured quantitatively in a constant known environment, free from tissue extracts which have long been known to have a profound yet unmeasurable effect upon the pulsation rate of excised myocardial tissue.

METHOD

Eggs of barred rock hens were incubated for the desired length of time and windows made in their blunt ends by the 
same technique described in a previous paper (Barry, '40). A coverslip was then sealed over the opening to prevent desiccation. An egg was placed in an observation incubator at $37.5^{\circ} \mathrm{C}$, and the rate of the heart beat was measured at intervals until it had remained constant for a quarter of an hour. After this constant rate and the developmental age had been determined, the entire embryo was removed to a dish containing Tyrode's solution at $37.5^{\circ} \mathrm{C}$. Here it was washed, and the entire thoracic region, with the heart attached, was removed by transverse cuts cephalic to the truncus arteriosus and caudal to the Ducts of Cuvier. Thus the heart was not injured, and retained its normal eurvature. This thoracic portion of the embryo was placed in an observation chamber through which flowed oxygenated Tyrode's solution of $\mathrm{pH} 7.7$, at a temperature of $37.5^{\circ} \mathrm{C}$.

Measurements were made of the rate of this excised heart, and it was then cut with irridectomy scissors into as many transverse segments as desired. In some cases the cut ends of the fragments were pinched together, but usually these surfaces reopened so that the lumen of the tube was patent. The pulsations of these fragments tended to attain a steady rate, often within 10 minutes of sectioning in the case of the more caudal levels of the heart. The anterior part of the ventricle, however, sometimes took as much as an hour and a half to reach this steady rate. The contractions were timed with a stopwatch during the period of acclimatization, while the final rates were recorded photographically by means of a moving film slit camera. This latter method was advantageous since it gave a permanent record of the pulsations from which the time for each successive beat could be determined. The recording device was in all essentials as described previously (Barry, '41).

Patten's ('22) illustrations were used as a basis of specifying the regions of the heart from which a given fragment was obtained. For the most part it was impractical to cut the same heart into more than six or seven pieces. The fragments were labeled according to the chambers they formed in the 
intact heart, i.e., sinus (S), atrium (A), ventricle (V). Following the nomenclature used by Kramer ('42) for the embryonic human heart, the tapering portion of the myocardial tube between the ventricle and the future site of the semilunar valves was designated the conus (C). That portion of the heart distal to the conus was called the truncus (T). Each chamber was considered to be made up of four transverse segments arranged along the antero-posterior axis of the cardiac tube. These segments were numbered one to four
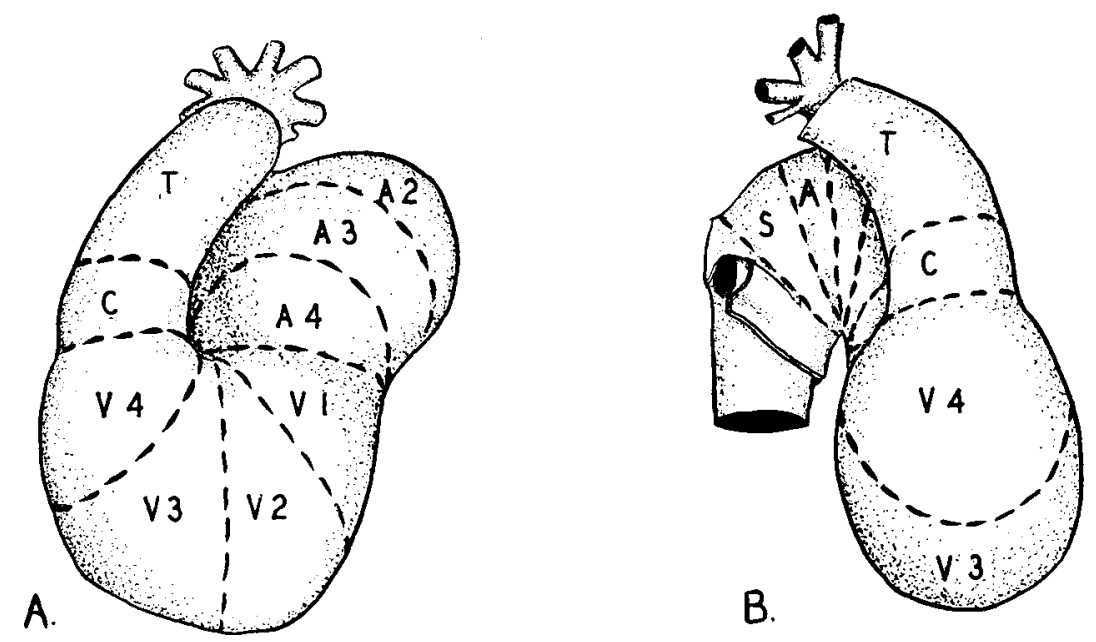

Fig. 1 Drawing of the heart of a 65 -hour chick embryo, showing the method of quartering its several chambers. A. Ventral view. B. Dextral view.

beginning with the most caudal; i.e., the most caudal quarter of the sinus would be designated S-1, while the next to the most cephalic quarter of the atrium would be $A-3$. Thus the heart could be subdivided into fourteen segments which could be labelled accurately as to their original location in situ (fig. 1). Although these myocardial fragments are quite minute, the recording device was sufficiently sensitive to record their pulsations.

\section{RESCLTS}

The photographic records from fragments of the heart of an 80-hour chick are shown in figure 2 . The vertical white 
lines on the records mark off intervals of $\frac{1}{3}$ second. It can be seen that the posterior end of the sinus, S-1, beat most rapidly at 154 per minute, while the posterior end of the atrium, A-1, beats less rapidly at 146 per minute. The posterior quarter of the ventricle, $\mathrm{V}-1$, beats still more slowly at 42 per minute. This gradient of inherent rate of pulsation between chambers can be designated as an intercameral gradient. In addition to this intercameral gradient there is also a gradient within each chamber which may be called intracameral. The intracameral gradient for the atrium can

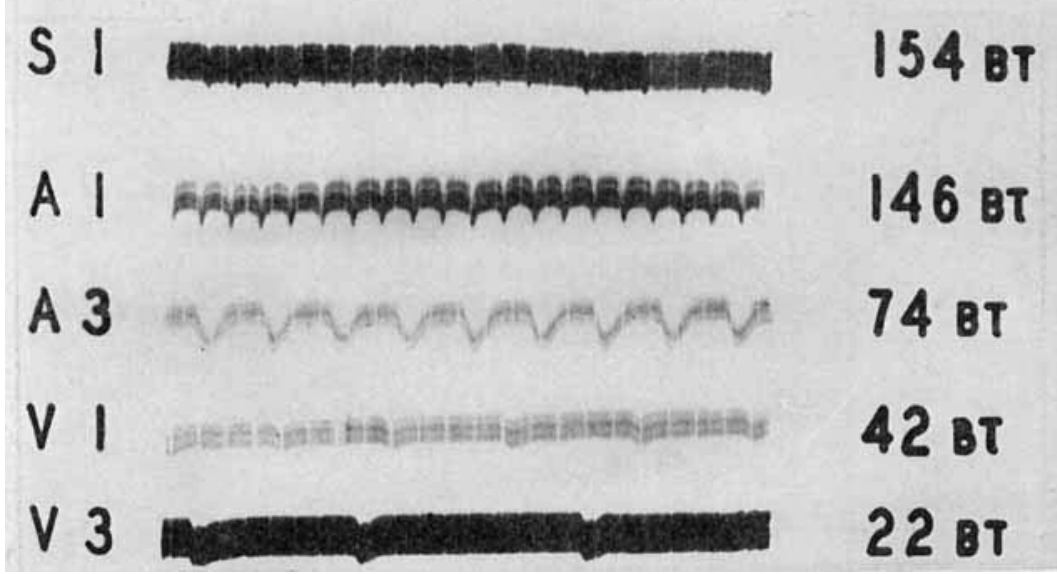

Fig. 2 Photographic recordings of the pulsations of heart fragments from an 80-hour chick embryo. The notations at the right indicate beats per minute.

be seen in figure 2, since the anterior half of the atrium, A-3, beats 74 times a minute, i.e., faster than $V-1$, and slower than A-1. Similarly V-3 beats more slowly than V-1, at 22 times a minute.

A point of interest is the fact that near the end of the record of S-1 the time between successive beats is precisely twice the "normal" interval. This indicates a 1-2 intrasinus heart block, which is a relatively common observation in these fragments from the caudal end of the heart. The possible significance of this will be treated below. 
Figure 3 illustrates the behavior of a typical heart from a 73-hour chick embryo when it is fragmented. The time for twenty beats of the intact heart in situ was found to be $6 \frac{1}{2}$ seconds. After a third measurement had shown this to be relatively constant, the omphalomesenteric vessels on both sides were cut open. $A$ indicates the time of this exsanguination. After the expected decrease in heart rate had been

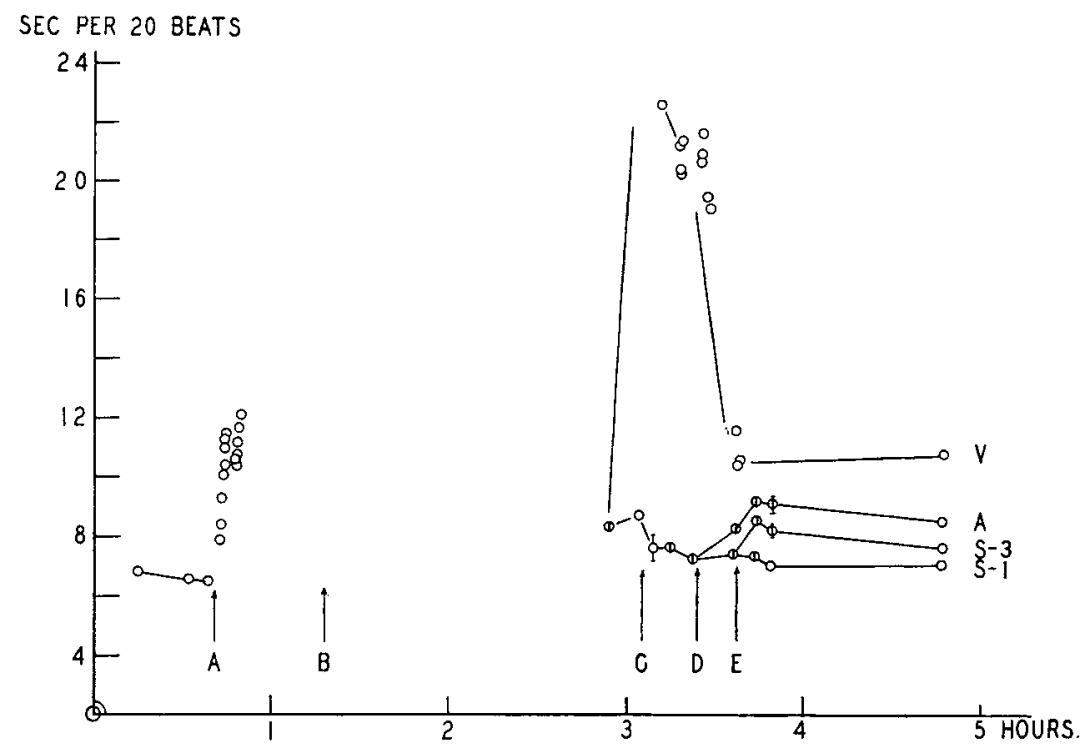

Fig. 3 Graph showing the rate of the entire heart, and of heart fragments from an embryo of 73 hours. Ordinate - inverse of rate (seconds per 20 beats). Abscissa - time in hours. A - time of eutting omphalomesenteric vessels in ovo. $\mathrm{B}$ - time of excision of thoracic region and removal to observation chamber. $\mathrm{C}$ - time of transection between atrium and ventricle. $\mathrm{D}$ - time of transection between atrium and sinus. $\mathrm{E}$ - time of transection between anterior and posterior halves of the sinus.

recorded (Barry, '41), the heart and thoracic region of the body was excised ( $\mathrm{B}$ on the graph), and transferred to the observation chamber. After about an hour its rate was again measured. At times indicated on the graph by $C, D$, and $E$, respectively, the heart was transsected between the atrium and ventricle (C); between the atrium and sinus (D); and 
between the anterior and posterior halves of the sinus $(\mathrm{E})$. A length of time was allowed to elapse between each sectioning sufficient to permit the fragments to attain a steady beat. It can be seen that the most posterior part of the heart (S-1) maintained an essentially constant frequency, while that of the more anteriorly situated regions was progressively less. From a series of similar measurements on other hearts of differing ages it was found that the rate of any portion of the myocardium was a function of its position along the axis of the cardiac tube. In other words any segment has a higher rate than the ones cephalic to it, and lower than those which lie caudal to it. It should be understood that, as used here, the terms cephalic and caudal designate relative positions along the original longitudinal axis of the embryonic cardiac tube, regardless of the torsions and displacement the heart may have undergone at any given stage.

This gradient was found to be both inter-and intracameral. In figure 4 the results from eight individuals are shown graphically. The rates of the several fragments of the individual hearts were expressed as percentages of the pacemaker rate. When these percentages were plotted as ordinates, against the antero-posterior axis of the heart as abscissa, histograms were obtained which expressed this gradient of inherent rhythmicity within the myocardial tube. It will be noted that a fragment is designated according to the level of its most caudal end, since this end will have the highest rate, and will consequently act as pacemaker for the whole fragment.

If the inherent rate of pulsation of a single region of the heart be followed during development, it can be seen to increase at first, and then slowly decrease with age. The regions labeled similarly in the present experiment may be considered comparable even though they come from hearts of different ages, since once they have appeared the sulci may reasonably be regarded as fixed with relation to the adjacent myocardium during development. In figure 5 the rates of the posterior 

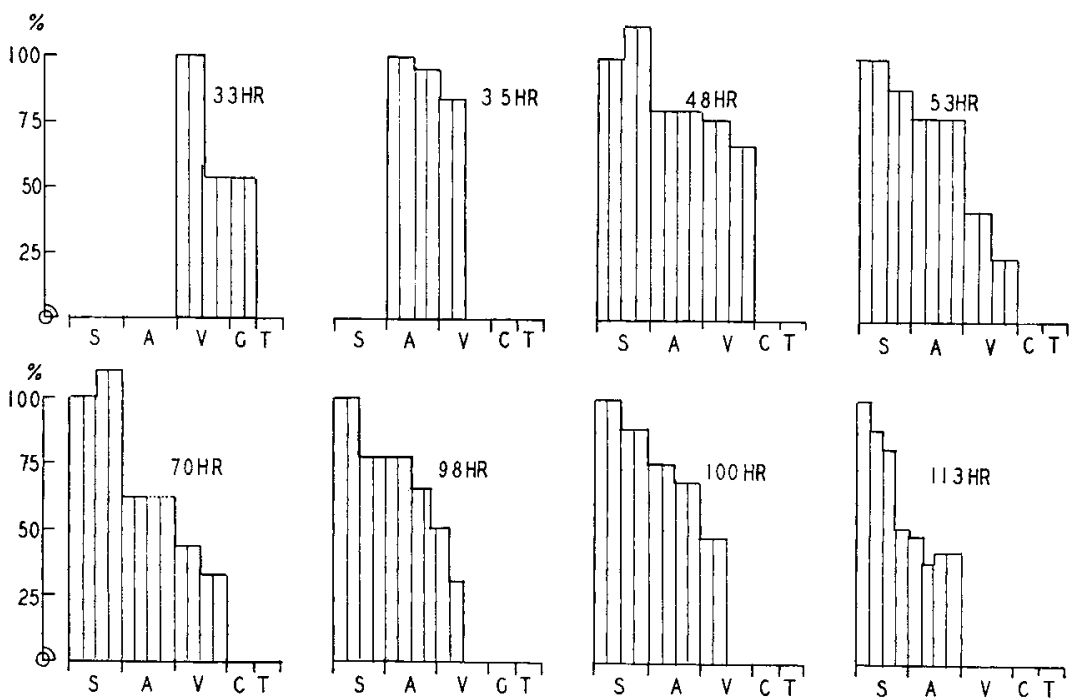

Fig. 4 Series of histograms showing gradient in the inherent rate of the myocardium along the cardiac tube at various ages. Ordinate - rates of pulsation expressed as per cent of the rate of the pacemaker. Abscissa-chambers of the heart arranged in cephalo-caudal sequence from right to left.

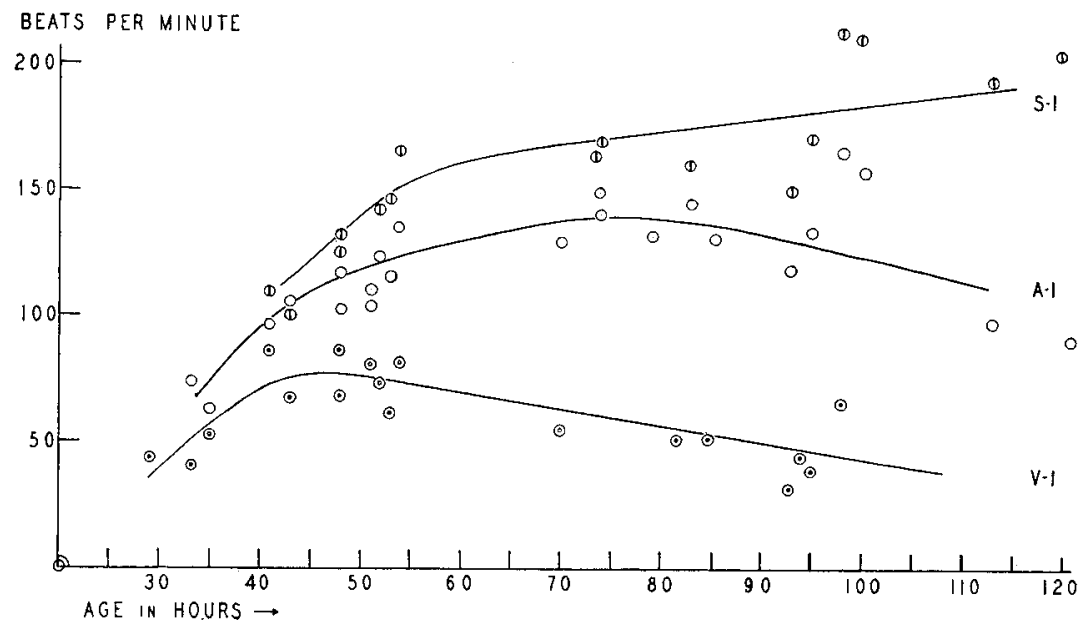

Fig. 5 Curves showing the inherent rate of rhythmicity of the caudal ends of the sinus ( $\mathrm{S}-1$ ), atrium (A-1), and ventricle ( $\mathrm{V}-1$ ) in embryos of various ages. Ordinate-rate in beats per minute. Abscissa-age of embryos in hours of incubation. 
parts of the ventricle ( $\mathrm{V}-1$ ), the atrium (A-1), and the sinus (S-1) have been plotted against the age of the embryo. The rate of V-1 is seen to decrease gradually, as does that of the atrium, while that of the pacemaker (S-1) parallels the rate of the intact heart. The curves describing the similar behavior of the anterior parts of these chambers have been omitted from this figure for the sake of clarity, but as a necessary consequence of the gradients shown in figure 4, would fall between the curves here shown. It is interesting, although not surprising, that these curves are very similar to the ones described by Copenhaver for the heart of Amblystoma.

When the entire hearts were excised it was found that in many cases a partial or complete heart block developed. This was found either between the atrium and ventricle or between atrium and sinus. Although this interference with the conduction of the contraction wave was most commonly seen in the region of the sulci, it was also seen in other regions of the heart. This phenomenon is to be expected in view of the fact that a decrease in pressure in the intact heart in situ caused a similar disruption of the myocardial conduction (Barry, '41). In one case when favorable illumination under the medium power of the microscope allowed particularly clear observation of the sinus of the excised heart from a 75-hour chick, the pacemaking function could be seen to migrate back and forth over the sinus in a caudo-cephalic direction. The interesting correlation here is that the rate of the entire heart exhibited a smoothly fluctuating character. This was such that when the pacemaker lay at the caudal end of the sinus (S-1) the heart rate was rapid, and as the pacemaker migrated forward to $\mathrm{S}-2$ or $\mathbf{S}-3$, the rate progressively declined. Up to the present I have not been able to duplicate the conditions necessary for the elicitation of this striking phenemenon. However, it does furnish one case of direct visual corroboration of an intrasinus block such as shown in the record of figure 2, S-1. 


\section{DISCUSSION}

From the observations of all the fragmentation experiments presented above, we can arrive at certain broad conclusions. In the first place, each of these bits of myocardium was treated similarly, and its rate of pulsation was measured after it had come to an equilibrium in a saline environment of fixed composition at a constant temperature. Since they were all measured under identical conditions, their rates of contraction were taken as direct measures of their inherent rhythmicity.

Thus from the above results it is possible to give a generalized account of a series of functional phases through which any segment of myocardium passes during its ontogeny.

During phase one the myocardium can be seen to form a part of the cardiac tube, but it does not yet possess the power of inherent rhythmicity, nor can it be stimulated to contract. The second phase is one during which the tissue starts to pulsate spontaneously, and exhibits an initially rapid but gradually lessening acceleration. The pulsations of a segment of the myocardium during this phase have, or at least soon attain, a higher rate of pulsation than the previously formed regions of the heart. It is during the first part of the second phase that any specific myocardial segment acts as the pacemaker.

Then follows a third phase, starting at the time when the pulsation frequency of the segment no longer accelerates. During this phase the inherent myocardial rate slowly decelerates, although the tissue in situ pulsates synchronously with the new pacemaker at a frequency higher than its own inherent rate. In the more anterior regions of the heart, this third phase is followed by a fourth, which is typified by the loss of the power of automaticity, although the tissue is still fully able to contract when appropriately stimulated either in situ or in vitro. These phases are certainly true for atrial and ventricular musculature, and it seems not improbable that the myocardium of the sinus passes through at least the three initial phases. However, there are at present inadequate data to insist upon this latter point. 
It is significant that the curve described by the excised pacemakers of the heart, i.e. S-1, at various ages follows quite closely the curve of the rate of the intact hearts (fig. 5). In other words, the pacemaker of an embryonic heart in this age range, when excised in Tyrode's solution, will attain a rate of pulsation very near to that which it exhibited in situ at the same temperature. This makes it seem likely that the initial rapid acceleration and subsequent slower increase in heart rate during the first 4 days of incubation may be regarded as due in great measure to the successive addition of new segments of myocardium of increasingly higher inherent automaticity.

These observations have not answered the fundamental problem as to why each successive addition to the heart has a higher rate. However, they do furnish adequate reason for believing that secondary factors such as blood pressure which might well have been expected to have had an influence upon the rate of pulsations of the pacemaker of the developing heart have, in all probability, a relatively minor effect on its rate of pulsation.

\section{SUMMARY}

The hearts of embryonic chicks of from 29 to 120 hours incubation age were transected into several fragments. The pulsations of these segments were recorded photographically, and their frequencies were measured in a constant environment of oxygenated Tyrode's saline solution at $37.5^{\circ} \mathrm{C}$.

A gradient of inherent rhythmicity was demonstrated both inter- and intracamerally such that any fragment of myocardium beat more rapidly than those segments lying anterior to it, and more slowly than those posterior to it along the axis of the heart.

Evidence was obtained of the occurrence of complete intracameral heart block. One ease was observed where the pacemaker function migrated back and forth along a pulsating myocardial fragment, with a resultant fluctuating pulsation frequency. 
The inherent rates of comparable levels of the heart were compared in terms of the age of the embryo. From these results it was possible to establish a series of functional phases through which any segment of myocardium passes during development:

Phase 1 -during which the myocardial segment does not contract spontaneously.

Phase 2-typified by the presence of a spontaneous beat which exhibits a gradually lessening acceleration.

Phase 3 -during which the inherent rate of the myocardium decelerates.

Phase 4-The myocardium of the more anterior levels of the heart passes into a fourth phase when it loses its automaticity, although in situ it can still contract synchronously with the rest of the heart.

\section{LITERATURE CITED}

BARRY, A. 1940 Age changes in the pulsation frequency of the embryonic ehick heart. J. Exp. Zool., vol. 85, pp. 157-170.

1941 The effect of exsanguination on the heart of the embryonic chick. J. Exp. Zool., vol. 88, pp. 1-15.

Cour, A. E. 1925 Physiological Ontogeny. A. Chicken Embryos. VI. Differentiation in the chicken embryo heart from the point of view of stimulus production. J. Exp. Med., vol. 42, pp. 299-310.

COPENHAVER, W. M. 1939 Initiation of beat and intrinsic contraction rates in the different parts of the Amblystoma heart. J. Exp. Zool., vol. 80, no. 2, pp. 193-224.

Johnstone, P. N. 1924 Studies on the Physiological Anatomy of the embryonic heart. I. The demonstration of complete heart block in chick embryos during the second, third and fourth days of incubation. Johns Hopkins Hosp. Bull., vol. 35, pp. 87-90.

KRAMER, T. C. 1942 The partitioning of the truncus and conus, and the formation of the septum membranaceum in the human heart. Doctoral thesis in the University of Michigan Library.

PatTeN, B. M. 1922 The formation of the cardiac loop in the ehick. Am. J. Anat., vol. 30, no. 3, pp. 373-397.

PatTen, B. M., AND T. C. KrAMER 1933 The initiation of contraction in the embryonic chick heart. Am. J. Anat., vol. 53, no. 3, pp. 349-375.

Pickering, J. W. 1893 Observations on the Physiology of the embryonic heart. J. of Physiol., vol. 14, pp. 383-466. 